\title{
Malassezia-Hefen: Bedeutung für das atopische Ekzem
}

\author{
Malassezia Yeasts: Significance for Atopic Dermatitis
}

\author{
Autor \\ P. Mayser \\ Institut \\ Zentrum für Dermatologie und Andrologie, Gießen
}

\section{Bibliografie}

DOI $10.1055 / \mathrm{s}-0028-1103463$

Online-Publikation: 25. 2. 2009

Akt Dermatol 2009; 35:

315-318 @ Georg Thieme

Verlag KG Stuttgart · New York ISSN 0340-2541

Korrespondenzadresse

Prof. Dr. Peter Mayser

Zentrum für Dermatologie und Andrologie

der Justus-Liebig-Universität

Gaffkystr. 14

35385 Gießen

Peter.Mayser@derma.med.

uni-giessen.de

\section{Zusammenfassung}

$\nabla$

Malassezia-Hefen als Bestandteile der residenten Flora der menschlichen Haut können auch Hauterkrankungen verursachen. So können sie zur chronischen Head-Neck-Dermatitis (HND) als einer Sonderform der atopischen Dermatitis beitragen. Hierfür sprechen neben dem Nachweis von Malassezia-spezifischen IgE-Antikörpern, der Prick-Testung und dem Atopie-Patch-Test insbesondere auch die Ergebnisse klinischer Studien, bei denen Antimykotika topisch oder systemisch

Malassezia (früher Pityrosporon)-Hefen gehören zur residenten Flora der menschlichen Haut und der vieler Warmblüter [1]. Derzeit werden 12 Spezies (M. furfur, M. sympodialis, M. globosa, M. pachydermatis, M. obtusa, M. restricta, M. sloofiae, M. dermatis, M. equi, M. japonica und M. yamatoensis) unterschieden, die mit Ausnahme von M. pachydermatis alle obligat lipiddependent sind. Sie finden sich daher vor allem in den talgdrüsenreichen Arealen der Haut. Ein Standardmedium zur Anzucht ist mdixon-Agar mit den Lipidquellen Tween 40 und Olivenöl, die Differenzierung erfolgt mit dem Tween-Auxanogramm und/oder über eine Sequenzierung der ITS1 der rDNA nach Makimura [2]. Auf gesunder Haut werden am häufigsten $M$. sympodialis und M. globosa nachgewiesen. Zu den häufigsten Malassezia-assoziierten Krankheitsbildern gehören die Pityriasis versicolor, das seborrhoische Ekzem und die Malassezia-Follikulitis. Die Pathogenese dieser Erkrankungen ist jedoch noch nicht vollständig geklärt, eine Spezies-spezifische Assoziation besteht nicht.

Neuere Untersuchungen zeigen, dass auch die Hautflora in der Pathogenese der atopischen Der-

\footnotetext{
* Vortrag beim 2. Wiesbadener Symposium Kinderderma-
} tologie, 12 . November 2008 bei der HND eingesetzt wurden. Inwieweit diese Ergebnisse auch auf die Behandlung von Kindern mit atopischen Ekzem übertragen werden können, ist derzeit unklar. Mit Einsetzen der Pubertät ändern sich Lipidmenge, Lipidzusammensetzung und auch die Malassezia-Besiedlung der Haut. Pathophysiologisch bedeutsam scheint nach den vorliegenden Daten ein molekulares Mimikry insbesondere gegenüber den Allergenen Mala S10 (heat shock protein) und S11 (manganese superoxide dismutase) zu sein, die eine hohe Homologie zu humanen Proteinen aufweisen.

matitis (AD) bedeutsam sein könnte [3]. Neben Staphyloccocus aureus betrifft dies insbesondere auch die lipophilen Malassezia-(früher Pityrosporum-) Hefen [4,5]. Aufgrund ihrer Verteilung könnten sie zur chronischen Head-Neck-Dermatitis (HND) als einer Sonderform der AD beitragen. Folgende Befunde stützen diese Vermutung: 1. Spezifisches IgE im Serum gegenüber Malassezia-Hefen ist in $20-100 \%$ bei Atopikern nachzuweisen, bei Erwachsenen mehr als bei Kindern sowie in besonderen Maße bei der Head-Neck-Dermatitis [6]. Kommerziell erhältliche Testallergene (Pharmacia ImmunoCAP) umfassen M. furfur (CBS 1878), M. sympodialis (CBS 7222) sowie M70 = „P. orbiculare" (ATCC 42132), welcher der Spezies M. sympodialis zugeordnet werden konnte. Ein weiterer Test besteht aus einer Allergenmischung von M. sympodialis, M. globosa und M. restricta (Pharmacia Immunocap m227). Rekombinante Allergene stehen kommerziell noch nicht zur Verfügung.

2. Der Prick-Test mit Malassezia-Extrakten ist positiv in $32-60 \%$ der Atopiker und korreliert nach vorliegenden Daten mit der Schwere und Lokalisation, jedoch sind auch hier keine standardisierten Reagenzien verfügbar. 


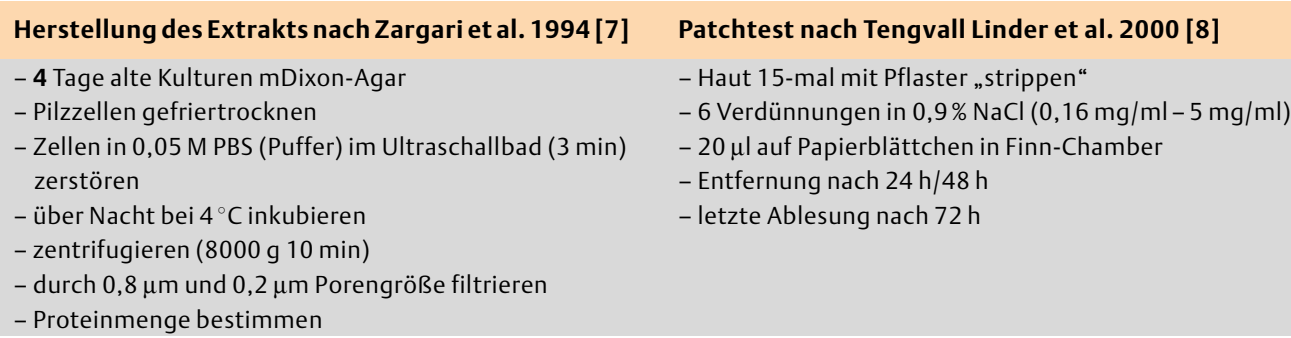

\section{Patchtest nach Tengvall Linder et al. 2000 [8]}

- Haut 15-mal mit Pflaster „strippen“

- 6 Verdünnungen in $0,9 \% \mathrm{NaCl}(0,16 \mathrm{mg} / \mathrm{ml}-5 \mathrm{mg} / \mathrm{ml})$

- $20 \mu$ l auf Papierblättchen in Finn-Chamber

- Entfernung nach 24 h/48 h

- letzte Ablesung nach $72 \mathrm{~h}$

Tab. 1 Herstellung und Durchführung des AtopiePatch-Tests mit MalasseziaHefen.

Tab. 2 Studien zur Wirksamkeit von Antimykotika bei der Head-Neck-Dermatitis [9-14].

\begin{tabular}{|c|c|c|c|c|}
\hline Autor & Charakter & $\mathbf{N}$ & Dosis/Zeit & \\
\hline Clemmensen 1983 & $\mathrm{Db}$, rand, $\mathrm{pc}$ & 19 & $\begin{array}{l}\text { Ketoconazol } 200 \mathrm{mg} / \mathrm{d} 4 \text { Wo. }(n=9) \text { vs. Placebo } \\
(n=10)\end{array}$ & $\begin{array}{l}\text { Signifikante Besserung bei Pat. mit HND, aber } \\
\text { nicht bei generalisierter AD }\end{array}$ \\
\hline Back 1995 & Offen & 20 & $\begin{array}{l}\text { Ketoconazol } 200 \text { mg/2 Monate; dann } 200 \text { mg } \\
2 \times / \text { Woche für } 3 \text { Monate }\end{array}$ & $\begin{array}{l}\text { Klinische Besserung }(p<0.01) \text {, Reduktion des } \\
\text { Malassezia-spezifischen } \lg E(p<0.05)\end{array}$ \\
\hline Broberg 1995 & $\mathrm{Db}$, rand & 53 & $\begin{array}{l}\text { Hydrocortison/Miconazol Creme topisch } 2 \times / d \\
\text { und Ketoconazol Shampoo } 2 \times / \text { Woche vs. } \\
\text { Hydrocortison Creme und Placebo Shampoo } \\
\text { über } 4 \text { Wo.; orales Flucloxacillin } 2 \text { Wo., Follow- } \\
\text { up } 6 \text { Wochen }\end{array}$ & $\begin{array}{l}\text { Signifikante Besserung in beiden Gruppen, } \\
\text { keine Differenz }\end{array}$ \\
\hline Back 2001 & $\mathrm{db}, \mathrm{pc}$, rand & 29 & $\begin{array}{l}\text { Ketoconazol } 200 \mathrm{mg} / \mathrm{d}(\mathrm{n}=15) \text { vs. Placebo } \\
(\mathrm{n}=14) \text { für } 3 \text { Monate, topische Steroide wenn } \\
\text { erforderl. }\end{array}$ & Besserung in beiden Gruppen \\
\hline Lintu 2001 & $\mathrm{db}, \mathrm{pc}$, rand & 80 & Ketoconazol 200 mg/d vs. Placebo 30 Tage & Signifikante Besserung in der Verum-Gruppe \\
\hline Svejgaard 2004 & $\mathrm{db}, \mathrm{pc}$, rand & 53 & $\begin{array}{l}\text { Itraconazol } 400 \text { mg/d, Itraconazol } 200 \text { mg/d } \\
\text { oder Placebo über } 7 \text { Tage; } 105 \text { Tage follow-up }\end{array}$ & $\begin{array}{l}\text { Signifikante Besserung mit } 200 \text { mg Itraconazol } \\
\text { nach } 14 \text { Tagen }\end{array}$ \\
\hline
\end{tabular}

3. Der Atopie-Patch-Test scheint mit dem spezifischen IgE zu korrelieren und ist positiv bei $24-64 \%$ der AD Patienten. Kommerziell ist der Test ebenfalls nicht verfügbar. Die Präparation des in der Literatur gebräuchlichsten Allergenextraktes sowie die Durchführung des Tests fasst $\bigcirc$ Tab. 1 zusammen. Als wichtigstes Argument für eine Bedeutung von Malassezia-Hefen in der Pathogenese der HND wurde die klinische Wirksamkeit von Antimykotika angesehen [9-14]. Während für die systemische Gabe (Ketoconazol, Itraconazol) mehrere Studien vorliegen, wurde für die topische Anwendung nur eine kontrollierte Studie publiziert. Eine Übersicht über die publizierten Studien gibt $\bigcirc$ Tab. 2 .

In einer eigenen Studie wurden 50 Patienten (21 Männer, 29 Frauen) mit einer zumindest seit 6 Monaten bestehenden mäßigen bis schweren HND in eine prospektive doppelblinde Studie aufgenommen [15]. Alle zeigten eine zumindest $10 \%$ ige Beteiligung der Head-Neck-Region ( $\bullet$ Abb. 1). Die Ausprägung der Erkrankung wurde mittels IGA (investigators global assessment), dem EASI-Score für die Head-Neck-Region sowie einer Juckreizskalierung bewertet. IgE-Antikörper gegen M. sympodialis und/ oder M. furfur zumindest der CAP-Klasse 1 waren Voraussetzung für eine Studienteilnahme. Die Patienten trugen entweder eine $1 \%$ ige Ciclopiroxolamin-Creme (Batrafen; Aventis Pharma, Bad Soden, FRG) oder die korrespondierende Basiscreme zweimalig täglich dünn über 28 Tage in den betroffenen Arealen auf. $16 \mathrm{~Pa}-$ tienten in der Verum-Gruppe und 14 Patienten in der PlaceboGruppe beendeten die Studie. Zur Untersuchung der Schweregrad-Veränderung des atopischen Ekzems wurden für alle Patienten die IGA-Differenzen zwischen den einzelnen Messzeitpunkten gebildet. Es zeigten sich signifikante Unterschiede zwischen Experimental- und Kontrollgruppe in der IGA-Score-Veränderung von Zeitpunkt $\mathrm{t} 3$ nach $\mathrm{t} 4$ sowie über den Gesamtzeitraum. Ähnliche, aber nicht signifikante Veränderungen finden

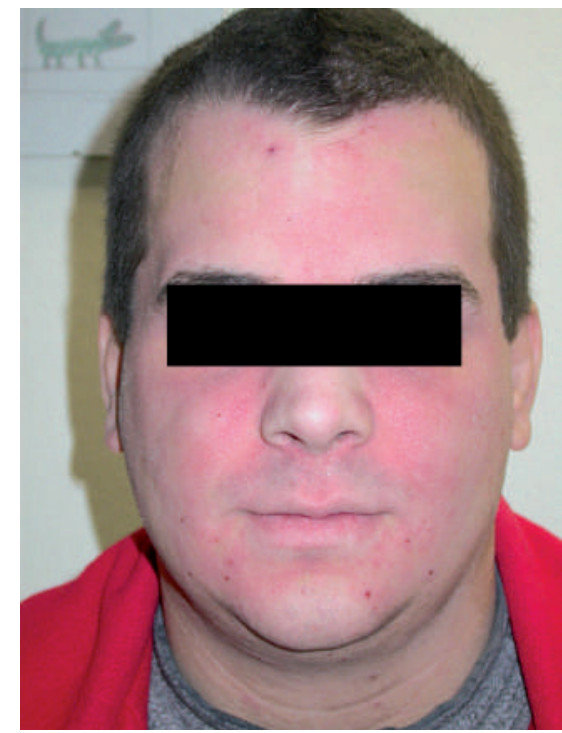

Abb. 1 Head-NeckDermatitis.

sich auch für die Werte des EASI, bezüglich der betroffenen Hautfläche und des Juckreizes. Beide Gruppen verbrauchten im Untersuchungszeitraum identische Mengen an Studienmedikation (Placebo-Gruppe: 86,1 $\mathrm{g} \pm 29,1$; Verumgruppe: 86,1 $\mathrm{g} \pm$ 25,5). Auch bezüglich des Einsatzes der weiteren Medikation (Dermatop, Dermatop-Basiscreme, -Basissalbe) ergaben sich keine signifikanten Verbrauchsunterschiede zwischen den beiden Gruppen. Es ließ sich somit ein günstiger Effekt einer topischen Antimykotika-Therapie auf die HND nachweisen.

Inwieweit diese Ergebnisse auch auf die Behandlung von Kindern mit atopischen Ekzem übertragen werden können, ist derzeit unklar. Mit Einsetzen der Pubertät ändern sich Lipidmenge, Lipidzusammensetzung und auch die Malassezia-Besiedlung 
Tab. 3 Derzeit bekannte Malassezia-Allergene [19].

\begin{tabular}{|c|c|c|c|}
\hline Allergen & Molekulargewicht & Homologie & Autor \\
\hline Mala s 1 & $37 \mathrm{kDa}$ & No homology with known proteinsmaltose-binding protein & Schmidt et al. 1997 \\
\hline Mala f2 & $\begin{array}{l}21 \mathrm{kDa} \text { (reduced) } \\
42 \mathrm{kDa} \text { (non-reduced) }\end{array}$ & $\begin{array}{l}\text { Peroxisomal membrane protein; putative Thioredoxin reductase } \\
\text { (C. boidinii)Aspergillus fumigatus allergen, Asp f3 }\end{array}$ & Yasueda et al. 1998 \\
\hline Mala f3 & $\begin{array}{l}20 \mathrm{kDa} \text { (reduced) } \\
40 \mathrm{kDa} \text { (non-reduced) }\end{array}$ & $\begin{array}{l}\text { Peroxisomal membrane protein; putative thioredoxin reductase } \\
\text { (C. boidinii)Aspergillus fumigatus allergen, Asp f3 }\end{array}$ & Yasueda et al. 1998 \\
\hline Mala f4 & $35 \mathrm{kDa}$ & Mitochondrial malate dehydrogenase & Onishi et al. 1999 \\
\hline Mala s 5 & $18 \mathrm{kDa}$ & Mala f2, Mala f3 & Lindborg et al. 1999 \\
\hline Mala s 6 & $17 \mathrm{kDa}$ & Cyclophilin (S. pombe) & Lindborg et al. 1999 \\
\hline Mala s 7 & $16 \mathrm{kDa}$ & No homology with known proteins & Rasool et al. 2000 \\
\hline Mala s 8 & $19 \mathrm{kDa}$ & No homology with known proteins & Rasool et al. 2000 \\
\hline Mala s 9 & $14 \mathrm{kDa}$ & No homology with known proteins & Rasool et al. 2000 \\
\hline Mala s 10 & $86 \mathrm{kDa}$ & Heat shock protein & Andersson et al. 2004 \\
\hline Mala s 11 & $22 \mathrm{kDa}$ & Manganese superoxide dismutase & Andersson et al. 2004 \\
\hline Mala s 12 & $67 \mathrm{kDa}$ & GMC oxidoreductase & Zargari et al. 2007 \\
\hline Mala s 13 & $13 \mathrm{kDa}$ & Thioredoxin & Limacher et al. 2007 \\
\hline
\end{tabular}

der Haut. Takahata et al. publizierten kürzlich eine vergleichende Analyse der Malassezia-Flora bei Erwachsenen und Kindern mit AD [16]. Malassezia restricta war prädominant bei Kindern, während bei Erwachsenen sowohl M. restricta als auch M. globosa vorherrschten. Im Vergleich zu Kindern zeigten Erwachsene eine erhöhte Reaktivität der IgE-Serumreaktionen gegen M. globosa und M. restricta. Lange et al. [17] untersuchten bei 141 Kindern mit AD im Alter von 3 bis 196 Monaten (Mittel 35,8, SD 38,4) die Sensibilisierung gegenüber 3 Malassezia-Arten (Pharmacia Immunocap m227). 58 Kinder waren bis zu 12 Monate, 83 über 12 Monate alt. Der SCORAD-Index betrug im Mittel 36 (Bereich 0-91, SD 20). Von den 141 Seren wurden 24 (17\%) positiv auf spezifisches IgE gegen Malassezia spp. getestet (Bereich $0,36-44,2 \mathrm{kU} / \mathrm{l})$. Neun von 58 Kindern $(15,2 \%)$ bis zu 12 Monaten waren positiv im Vergleich zu 15 von 83 (18,1\%) in der Gruppe der Älteren. Der jüngste sensibilisierte Patient war 4 Monate alt. Das Risiko einer Sensibilisierung gegenüber Malassezia korrelierte signifikant mit dem Gesamt-IgE-Wert $(r=0,510$, $P<0,0001)$ und schwach mit dem SCORAD Index $(r=0,24$, $P>0,005)$. Es zeigte sich keine signifikante Korrelation zwischen Malassezia-Sensibilisierung und Alter, Dauer oder Zeitpunkt des Einsetzens der Erkrankung. Eine Analyse des entsprechenden klinischen Bildes zeigte eindeutig, dass bei Patienten mit hohen IgE-Werten und schwerem klinischen Verlauf das Risiko einer Malassezia-Sensibilisierung höher liegt. Ob diese MalasseziaSensibilisierung eine pathogenetische Bedeutung hat oder ob sie Ausdruck einer gesteigerten Reagibilität ist, konnte aus der vorliegenden Untersuchung nicht abgeleitet werden.

Für eine Optimierung dieser therapeutischen Option „antimykotika bei AD“ wären neben der Auswahl geeigneter Patienten über Bestimmung der Pilzlast, des spezifischen IgE, des Prick-Testes und insbesondere des Atopie-Patch Testes gegenüber (rekombinanten) Malassezia-Antigenen auch eine Kombinationstherapie mit Antimykotika-haltigen Shampoos und/oder bei schweren Verläufen mit einer kurzzeitigen systemischen antimykotischen Therapie möglich.

Pathophysiologisch bedeutsam scheint nach den vorliegenden Daten ein molekulares Mimikry zu sein. Insbesondere die Allergene S 10 (heast shock protein) und S 11 (manganese superoxide dismutase) weisen eine hohe Homologie zu humanen Proteinen auf [18]. Der Grad der Sensibilisierung korreliert zur Krankheitsaktivität. Eine weitere Analyse der Malassezia-Allergene (Mala, derzeit 13; Tab. 3), ihre Zuordnung zu einzelnen Spezies sowie der Einsatz rekombinanter Allergene in der Allergie-Diagnostik könnte die Bedeutung der Malassezia-Hefen für die Pathogenese der AD und insbesondere der HND weiter abklären.

\section{Abstract}

\section{Malassezia Yeasts: Significance for Atopic Dermatitis} $\nabla$

Belonging to the resident flora of human skin, Malassezia yeasts can also cause skin diseases. They may contribute to the development of chronic head-neck dermatitis (HND) which is a special form of atopic dermatitis. This is supported by the demonstration of Malassezia-specific IgE antibodies, prick-testing and atopy patch test, but in particular by the results of clinical studies in which antimycotics had been applied topically or systemically for treatment of HND. It remains to be clarified whether these results are also applicable for children with atopic eczema. The onset of puberty is associated with changes in the amount and composition of lipids and increased Malassezia colonization of the skin. Available data indicate the pathophysiological significance of a molecular mimicry, especially of the allergens Mala S10 (heat shock protein) und S11 (manganese superoxide dismutase), which are highly homologous to human proteins.

\section{Literatur}

1 Hort $W$, Nilles $M$, Mayser $P$. Malassezia yeasts and their signifcance in dermatology. Hautarzt 2006; 57: 633-645

2 Makimura K, Tamura Y, Kudo M et al. Species identification and strain typing of Malassezia species stock strains and clinical isolates based on the DNA sequences of nuclear ribosomal internal transcribed spacer 1 regions. J Med Microbiol 2000; 49: 29-35

3 Baker BS. The role of microorganisms in atopic dermatitis. Clin Exp Immunol 2006; 144: 1 -9

4 Scheynius A, Johansson C, Buentke E et al. Atopic eczema/dermatitis syndrome and Malassezia. Int Arch Allergy Immunol 2002; 127: $161-169$

5 Schmid-Grendelmeier P, Scheynius A, Crameri R. The role of sensitization to Malassezia sympodialis in atopic eczema. Chem Immunol Allergy 2006; 91: 98 - 109

6 Mayser P, Gross A. IgE antibodies to Malassezia furfur, M. sympodialis and Pityrosporum orbiculare in patients with atopic dermatitis, seborrheic eczema or pityriasis versicolor, and identification of respective allergens. Acta Derm Venereol 2000; 80: 357-361 
7 Zargari A, Harfast B, Johansson S et al. Identification of allergen components of the opportunistic yeast Pityrosporum orbiculare by monoclonal antibodies. Allergy 1994; 49: 50-56

8 Tengvall Linder M, Johansson C, Scheynius A, Wahlgren C. Positive atopy patch test reactions to Pityrosporum orbiculare in atopic dermatitis patients. Clin Exp Allergy 2000; 30: 122 -131

9 Clemmensen OJ, Hjorth $N$. Treatment of dermatitis of the head and neck with ketoconazole in patients with type I sensitivity to Pityrosporum orbiculare. Semin Dermatol 1983; 2: 26-29

10 Back O, Scheynius A, Johansson SGO. Ketoconazole in atopic dermatitis: therapeutic response is correlated with decrease in Serum IgE. Arch Dermatol Res 1995; 287: $448-451$

11 Back O, Bartosik J. Systemic ketoconazole for yeast allergic patients with atopic dermatitis. JEADV 2001; 15: 34-38

12 Lintu P, Savolainen J, Kortekangas-Savolainen $O$ et al. Systemic ketoconazol is an effective treatment of atopic dermatitis with IgE-mediated hypersensitivity to yeast. Allergy 2001; 56: $512-517$

13 Svejgaard E, Larsen P, Deleuran $M$ et al. Treatment of head and neck dermatitis comparing itraconazole $200 \mathrm{mg}$ and $400 \mathrm{mg}$ daily for 1 week with placebo. JEADV 2004; 18: 445-449
14 Broberg A, Faergemann J. Topical antimycotic treatment of atopic dermatitis in the head/neck area. A double-blind randomized study. Acta Derm Venereol 1995; 75: 46 - 49

15 Mayser P, Kupfer J, Nemetz D et al. Treatment of head and neck dermatitis with ciclopiroxolamine cream - results of a double-blind, placebo-controlled study. Skin Pharmacol Physiol 2006; 19: 153-158

16 Takahata Y, Sugita T, Kato H et al. Cutaneous Malassezia flora in atopic dermatitis differs between adults and children. Br J Dermatol 2007; 157: $1178-1182$

17 Lange L, Alter N, Keller Tet al. Sensitization to Malassezia in infants and children with atopic dermatitis: prevalence and clinical characteristics. Allergy 2008; 63: 486 - 487

18 Schmid-Grendelmeier P, Fluckiger S, Disch $R$ et al. IgE-mediated and T cell-mediated autoimmunity against manganese superoxide dismutase in atopic dermatitis. J Allergy Clin Immunol 2005; 115: $1068-$ 1075

19 Mayser P, Lang S, Hort W. Pathogenicity of Malassezia Yeasts. In: Brakhage A, Zipfel P, Hrsg. The Mycota VI: Human and animal relationships. 2. Aufl. Heidelberg-New York: Springer Verlag, 2008: 115 - 154 